\title{
HÖLDER AND MINKOWSKI TYPE INEQUALITIES WITH ALTERNATING SIGNS
}

\begin{abstract}
Petr Chunaev
Abstract. We obtain new inequalities with alternating signs of Hölder and Minkowski type.

Mathematics subject classification (2010): 26D15.

Keywords and phrases: Alternating signs, Hölder and Minkowski type inequalities.
\end{abstract}

\section{REFERENCES}

[1] E. Beckenbach and R. Bellman, Inequalities, Springer-Verlag, Berlin, 1961.

[2] R. Bellman, On an inequality due to Weinberger, Amer. Math. Monthly 60 (1953), 402.

[3] R. Bellman, On inequalities with alternating signs, Proc. Amer. Math. Soc. 10 (1959), 807-809.

[4] Y. Benyamini And J. Lindenstrauss, Geometric Nonlinear Functional Analysis. Vol. 1, AMS Colloquium Publications, 48, American Mathematical Society, Providence, RI, 2000.

[5] M. BIERNACKI, Sur des inégalités remplies par des expressions dont les termes ont des signes alternés, Ann. Univ. Mariae Curie-Skłodowska. Sect. A. 7 (1953; 1954), 89-102.

[6] M. BJELICA, Refinement and converse of Brunk-Olkin inequality, J. Math. Anal. Appl. 227, 2 (1998), $462-467$.

[7] L. Bougoffa, On Minkowski and Hardy integral inequalities, J. Inequal. Pure Appl. Math. 7, 2 (2006), Article 60, 3 pp.

[8] H. D. BRunK, On an inequality for convex functions, Proc. Amer. Math. Soc. 7 (1956), 817-824.

[9] R. E. Castillo AND E. TROusselot, Reverse generalized Hölder and Minkowski type inequalities and their applications, Bol. Mat. (N.S.) 17, 2 (2010), 137-142.

[10] G. H. Hardy, J. E. LitTlewood, ANd G. Pólya, Inequalities, Cambridge University Press, Cambridge, 1952.

[11] E. LifLyAND AND S. TiKHONOv, A concept of general monotonicity and applications, Math. Nachr. 284, 8-9 (2011), 1083-1098.

[12] B. Mond And J. E. PeČARIĆ, Szegó and related inequalities for operator-convex functions, Soochow J. Math. 22, 1 (1996), 33-37.

[13] G. SZEGŐ, Über eine Verallgemeinerung des Dirichletschen Integrals, Math. Z. 52 (1950), 676-685.

[14] I. Olkin, On inequalities of Szegö and Bellman, Proc. Natl. Acad. Sci. USA. 45 (1959), 230-231; addendum, 1553.

[15] J. E. PeČARIĆ, On an inequality of G. Szegó, J. Math. Anal. Appl. 158, 2 (1991), 349-351.

[16] J. F. STEFFEnSEN, Bounds of certain trigonometrical integrals, C. R. Dixième Congrès Math. Scandinaves (1946), 181-186, Jul. Gjellerups Forlag, Copenhagen, 1947.

[17] O. VAlero, Quotient normed cones, Proc. Natl. Acad. Sci. India 116, 2 (2006), 175-191.

[18] H. F. Weinberger, An inequality with alternating signs, Proc. Natl. Acad. Sci. USA. 38 (1952), 611-613.

[19] E. M. Wright, An inequality for convex functions, Amer. Math. Monthly 61 (1954), 620-622.

[20] Y. D. ZhuAnG, On inverses of the Hölder inequality, J. Math. Anal. Appl. 161, 2 (1991), 566-575. 